\title{
New Perspectives for Workflow Analysis in the Health Italian Sector through Discrete Event Simulation: The Case of a Department of Laboratory Medicine
}

\author{
Adriano Torri' ${ }^{1}$, Oscar Tamburis ${ }^{2}$, Teresa Abbate ${ }^{1}$, Alessandro Pepino \\ ${ }^{1}$ Department of Electrical Engineering and Information Technologies, University Federico II, Naples, Italy \\ ${ }^{2}$ Department of Veterinary Medicine and Animal Productions, University Federico II, Naples, Italy \\ Email: torri2005@libero.it
}

Received 30 December 2014; accepted 27 April 2015; published 30 April 2015

Copyright (C) 2015 by authors and Scientific Research Publishing Inc.

This work is licensed under the Creative Commons Attribution International License (CC BY).

http://creativecommons.org/licenses/by/4.0/

(c) (i) Open Access

\begin{abstract}
The management systems currently used in the Italian healthcare sector provide fragmented and incomplete information on this system and are generally unlikely to give accurate information on the performances of the healthcare processes. The present paper introduces a combined discrete event simulation (DES)/business process management (BPM) approach as innovative means to study the workflow of the activities within the Department of Laboratory Medicine of the "San Paolo" Hospital in Naples (Italy). After a first "As-Is" analysis to identify the current workflows of the system and to gather information regarding its behaviour, a following DES-based "What-If" analysis is implemented to figure out alternative work hypotheses in order to highlight possible modifications to the system's response under varying operating conditions and improve its overall performances. The structure of the simulation program is explained and the results of the scenario analysis are discussed. The paper starts with a brief exploration of the use of DES in healthcare and ends with general observations on the subject.
\end{abstract}

\section{Keywords}

Healthcare Management, Performance Evaluation, Discrete Event Simulation, Business Process Management, Department of Laboratory Medicine

\section{Introduction}

Italian healthcare organizations operate in a dynamic context, characterized by considerable organizational and

How to cite this paper: Torri, A., Tamburis, O., Abbate, T. and Pepino, A. (2015) New Perspectives for Workflow Analysis in the Health Italian Sector through Discrete Event Simulation: The Case of a Department of Laboratory Medicine. Intelligent Information Management, 7, 93-106. http://dx.doi.org/10.4236/iim.2015.73009 
technological complexity. Accordingly, managers should rely on management systems in order to: (i) appropriately meet the operational needs of the structure they supervise; (ii) find out the most suitable solutions to accomplish the objectives defined in the business plan [1].

The present work aims at introducing a simulation-based operational paradigm [2] [3] that, along with classical management control systems, reproduces the behaviour of a complex structure [4]-[6], in order to provide useful real-time indicators to improve the quality of the management control [7]. The case study examined relates to the Department of Laboratory Medicine of the "San Paolo" Hospital in Naples (Italy), whose management methodology suffered from:

- staleness of data: the results of the business management activities were tied to a specific period in the past, corrective actions were based on past information and it was difficult to get real-time feedback regarding the effectiveness of such actions;

- weakness of stored data: the stored data were often incomplete, inaccurate and not related to the work activities carried out within the health service. Analyses of these data did not provide correct information.

The purpose was therefore to provide alternative workflows to be compared with those already performed in the department in order to improve the overall performances [8] [9].

\section{Materials and Methods}

In this paper, the discrete event simulation (DES) [10]-[14] technique is implemented, as support of the Business Process Management (BPM) [15] methodology, to study and improve the performances of healthcare facilities.

The DES is a simulation technique developed in the 1960s as part of industrial engineering and operations research dynamics, aimed at analysing and improving business processes. The use of simulation techniques has rapidly grown in recent years in the health sector, especially as for:

- patient-flow management;

- management of resources and correlated activities;

BPM includes all the activities required to create, organize, manage and improve the business processes of a company, with the aim of creating a process geared to make efficient and effective business; in other words, it means developing the ability to analyse, manage and adapt in real-time mode a set of business operations, IT applications and people, according to a structured sequence of phases, to achieve a common goal. Deploying a DES-based simulation system makes thus possible to both represent the real system from different points of view, and to analyse the behaviour and the characteristics of this system. The model so obtained is a representation of the reality built to answer specific questions, in a time faster than real, and its study leads eventually to solutions whose reliability increases with the accuracy of the model itself.

The use of simulation in the health sector has growth in the last years, and nowadays, especially in UK and USA, the use of this technique has become a popular approach for problem solving in the health sector. In Italy the use of this technique, especially coupled with the BPM methodology, is far from being well known, especially in the health sector.

Applying the BPM to the health sector allows: 1) increasing the value while reducing investment; 2) reducing management costs; 3 ) responding to regulatory requirements and arranging the processes according to the rules dictated by the new regulations; this becomes possible by means of:

- process modelling: means the formalization and implementation of the process through an application that leads to the simulation of the organizational work activities and the actual process;

- integration: means the ability used by the application/software to interact with a variety of management systems based on various platforms;

- monitoring: is the ability of the application/software to both coordinate the processes and maintaining a continuous control on what is happening;

- optimization and creation of a company-oriented process: refers to the ability of the application/software to improve the (desired) performances of the system.

The organization of a healthcare facility is a very complex matter and the use of both the BPM-based management policy and the DES technique offers the possibility to achieve the goals of a company/department/ structure featuring lower levels of complexity. An "As-Is" analysis performed through the use of DES allows implementing the actual workflows of the analysed system in a well-defined, structured and clear way. The DES approach founds on the fact that assumptions are independent from the model and can be generally administered 
at any time or resized in a probabilistic manner: this enables users to make a systematic examination of their assumptions, rather than guiding them in the construction of the model and the solution to the problem; the definition and the use of the "What-If" analyses, as cornerstone of the BPM, opens furthermore to the possibility of displaying new operational/functional scenarios, alternative to those already defined through the "As-Is" analysis. The new scenarios aim at achieving solutions that allow a qualitative and quantitative improvement of the performances of the system. To this purpose, the use of the DES approach helps to compare various strategies in order to identify the one that best fulfils the criteria pointed out from the decision makers.

\section{Model Design}

In this paper the workflow of the activities within the Department of Laboratory Medicine (DLM from now on) of the "San Paolo" Hospital in Naples (Italy) was studied through the use of the DES and the BPM. The "As-Is" analysis made possible to identify the current workflows of the system and to gather accordingly information regarding its behaviour; the "What-If" analysis was then helpful to figure out alternative work hypotheses in order to both highlight possible modifications to the system's response under varying operating conditions and improve its overall performances. The purposes of the work were:

- defining, under analytical and rigorous conditions, the operations performed in the DLM;

- obtaining information regarding the workflow never obtained before;

- identifying clearly both strengths and weaknesses of the system;

- proposing alternative solutions regarding the organization of the working activities in order to both highlight the response to changes and improve the performances of the DLM.

The latter point allowed especially figuring out a series of changes to the current working conditions: the scope was to evaluate by means of the simulation approach, the necessary interventions to carry out the requested modifications, and compare the results obtained with the outputs from the current working conditions. The analysis performed provided quantitative data to understand whether or not to adopt one of the tested solutions. These objectives can be achieved through the identification and the analysis of some "key performance indexes" (KPIs) that made possible to understand how the system evolves over time and what the responses to changes of the environment are. The KPIs are basically identified as follows (see Table 1).

\subsection{Characteristics of the Activities of the DLM}

The DLM is organized into seven sections, each one hosting different kind of operators and performing different types of exams. Table 2 shows the DLM working activities and the resources involved.

The sections operate in parallel; the weekly working hours are 36 for the technicians and 38 for the doctors. The Medical Chief performs only a supervising activity.

The working activities can be divided in: 1) "Routine Activities", organized on a single daily shift of 6 hours, for 7 days per week; 2) "Urgent Activities", carried-out with a 24/7 mode.

The acceptance of the requests of examination is a preliminary stage to the set of activities performed in the DLM; Table 3 shows how this activity is organized, depending on the type of requests received.

Each request is associated with one or more samples; each request module contains information about:

Table 1. KPIs of the model.

\begin{tabular}{ll}
\multicolumn{1}{c}{ Type } & \multicolumn{1}{c}{ KPI } \\
\hline Qualitative \& Quantitative KPIs & Number Completed Jobs \\
& Total Number of Each Type of Exam \\
Timing KPIs & \% of Usage of the Human Resource \\
& Average Time in System \\
Costs KPIs & Cost per Exam \\
& Total Cost both for Resources and Exams \\
& Cost of Each Human Resource \\
\hline
\end{tabular}


Table 2. Organization of the DLM working activities and resources involved.

\begin{tabular}{|c|c|c|c|c|c|}
\hline Type of Activities & Section Name & Resource Type & Role & Working Activities & $\mathbf{N}^{\circ}$ Resources \\
\hline \multirow{9}{*}{ Routine Activities } & \multirow{2}{*}{ Clinical Chemistry (CC) } & techn_cc & Technician & Clinical chemistry tests & 3 \\
\hline & & doctor_cc & Doctor & Clinical chemistry tests & 2 \\
\hline & \multirow{2}{*}{ Bacteriology (BA) } & techn_ba & Technician & Bacteriology tests & 2 \\
\hline & & doctor_ba & Doctor & Bacteriology tests & 3 \\
\hline & Serology (SI) & doctor_si & Doctor & Serology tests & 1 \\
\hline & Hormones Section (SO) & doctor_so & Doctor & Hormones tests & 2 \\
\hline & Allergy (AL) & doctor_al & Doctor & Allergy tests & 1 \\
\hline & OPF Section (OP) & techn_qp & Technician & Serum Electrophoresis tests & 1 \\
\hline & & doctor_qp & Doctor & Serum Electrophoresis tests & 2 \\
\hline \multirow{3}{*}{ Urgent Activities } & \multirow{2}{*}{ ER Section (SU) } & techn_su & Technician & Urgent Examinations & 1 \\
\hline & & doctor_su & Doctor & Urgent Examinations & 1 \\
\hline & Medical Chief & & Doctor & Supervision of laboratory activities & 1 \\
\hline
\end{tabular}

Table 3. Type of request, origin of the request and delivering-time of the requests.

\begin{tabular}{cccc}
\hline Type of Request & Origin of the Request & Days & Timing \\
\cline { 3 - 4 } Non-Urgent & Internal & Monday-Saturday & 08.00 A.M. to 10.30 A.M. \\
Urgent & External & Monday-Saturday & 11.00 A.M. to 12.00 A.M. \\
\hline
\end{tabular}

- Patient demographic data;

- Information about the requesting department;

- Information about the requesting physician;

- Information about the suspected diagnosis.

Blood samples are transported into tubes closed with a stopper. Samples of other nature are transported via different containers (e.g. plastic container or swabs with transport medium). The type of examination to be executed on the blood samples was defined according to the colour of the cap of the tube in which the sample was delivered.

\subsection{Building the Simulation Model}

The first step toward the workflow analysis consisted in the direct observation of the activities at the DLM, and lasted about six months. A survey administration to the staff was then performed, which led to gathering data about:

- Role of staff;

- Responsibilities of staff;

- General comments;

- Details of any critical issues.

After this first step, information was acquired about working activities, and related timings, carried-out in the unit.

Having set up a seven-day-period for the simulation time, information was acquired about working activities, and related timings, carried out in the unit during the week going from 01/10/2012 to 07/10/2012, since the data gathered from the workload dynamics provided a good representation of the typical DLM workloads. The data 
stored in the database of the Laboratory Information System (LIS) implemented in the DLM were eventually collected, which included:

- Information on examinations performed;

- Information on patients treated;

- The department that requested the examination;

- Date and time of the examination requested;

- List of the examinations prescribed.

From these files the following information could be deduced:

- Data from requests for routine examination (internal);

- Number of requests for urgent examination;

- Data from requests for routine examination (external).

These results were integrated with those coming from both the direct observations of the workflow and the questionnaires submitted to the staff. This was necessary since each single information flow on its own could not enable us to build a complete model. The descriptive diagram (direct observations, data gathering from ITsystems and questionnaires) is shown in Figure 1.

The events by which work activities carried out have been defined are:

- The instants of occurrence of the requests;

- The type of tests to be performed.

Files obtained from the data input took into account:

- Exams addressing: 1) Routine Activities; 2) Urgent Activities;

- Group requests by department;

- Macro-Areas in which examinations are performed.

The division between "routine activities" and "urgent activities" was necessary because the workflow of routine activities was the only one considered for the analysis: routine activities, both for the amount of work and the type of work, were illustrative of the functioning of the system under examination. In order to get to a proper modelling of the routine workflow activities, the input data must be divided into two files, depending on the origin of the request: internal (departments) or external. All examinations were classified into 15 macro-areas, whereas the data related to examinations were linked to the request of laboratory tests through a so-called Examination Type Label using: 1) 0 (examination not required); 2) other than 0 (examination required).

The designing of the model was performed by means of the SIMUL8 simulation software [16] and relied upon the mentioned 15 macro-areas (or branches), which operated in parallel, departing from the acceptance of the samples up to the full reporting. Files. csv (comma separated values) were used to load the data, featuring:

- Examination request ID Label;

- Time Label (means the actual timing of arrival of the requests to the laboratory);

- Examination Type Labels;

- Sample Size Label (means the number of samples each individual request is comprised of).

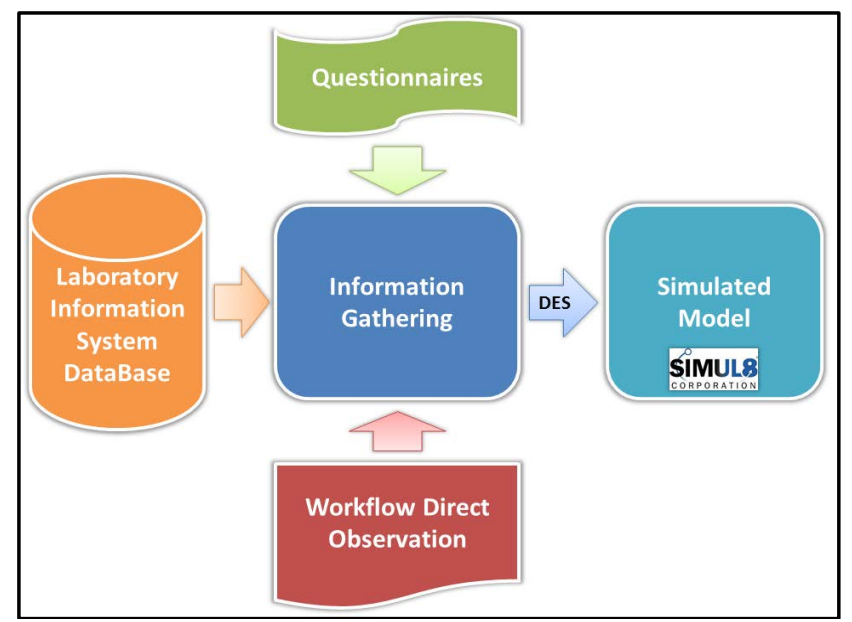

Figure 1. Information gathering workflow. 
For each single token (i.e. the single working resource) an arrival time is defined. This value is defined using a label, named "Time", contained in the .csv file. This file is uploaded at every simulation run. The values used in the .csv file were obtained from the LIS.

Figure 2 depicts the flowchart of the activities of the DLM, designed with SIMUL8; it has two entrances, featuring as the starting points of the model: one concerning the departments (both outpatients from ER and inpatients), and the other concerning the surgery (meaning inpatients that need more specific exams before undergo surgical interventions).

The simulation model realized with the software is instead presented in Figure 3.

\subsection{Workflow Activities}

\subsubsection{From the Requests to the Execution of the Exams}

As introduced in Figure 4, the input.csv file determines the arrival time of the requests. In this phase, the operators of the CC group (see Table 2) verify the correspondence between the type of request made and the type of the test-tubes received. In case of correspondence the request is accepted and it is possible to proceed with the examination, otherwise a note will be placed in the file relating to the request, indicating the reason why the examination was not performed.

The sorting of the tokens was obtained through an operation of batching. This action is performed in the routing-out (output stage) of the "samples split" activity. After these first steps the samples will be sorted in the mentioned 15 branches of the flow. For some type of exams (Allergy, Base, Coagulation, Hormones, Protein Panel, and Serology tests) after the sorting stage, there is a need for a centrifugation phase, as depicted in Figure 5. This activity is required to prepare the tubes for the subsequent examinations. During this phase the tubes are centrifuged for 6 minutes, and then addressed to the various branches of the model, in order to continue with the examinations.

The "accumulation of samples" and "sample preparation" steps have common characteristics for all the examinations types, an example of which is shown in Figure 6.

The following phase of "exams execution" is regulated according to the Laboratory daily working plan. The daily number of each type of test varies according to the number of the daily incoming requests (see Table 4).

\subsubsection{From the Results to the Final Reporting}

The following results of the analyses are referred to both hospitalized and external patients: in the first case, the results are compared with those previously stored in the databases of the facility in order to assess the evolution of the health status of the patient; in the second case, the results are studied, validated and then transmitted to the information system for the final reporting - the final stage of the system. The timing of the reporting activities differs, depending on the type of examination performed, as shown in Table 5.

\section{Validation and Analysis of the Model}

The model created with the SIMUL8 software was then validated by means of a two-step method, articulated in: 1) formal validation; 2) structural validation.

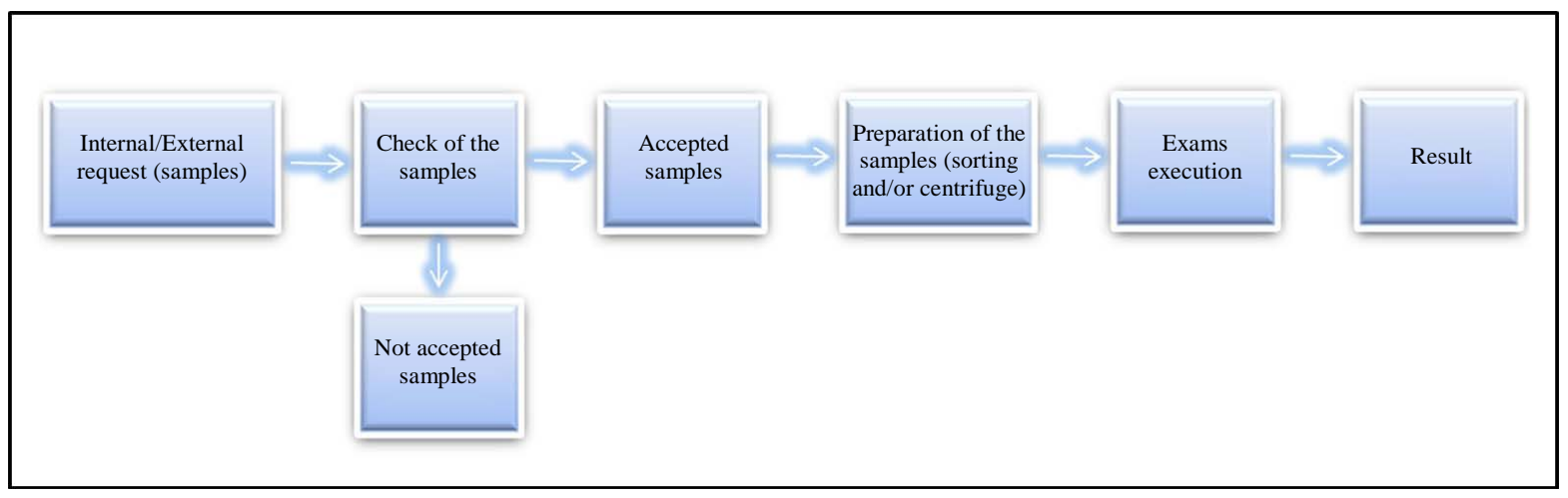

Figure 2. Flowchart of the activities of the DLM. 


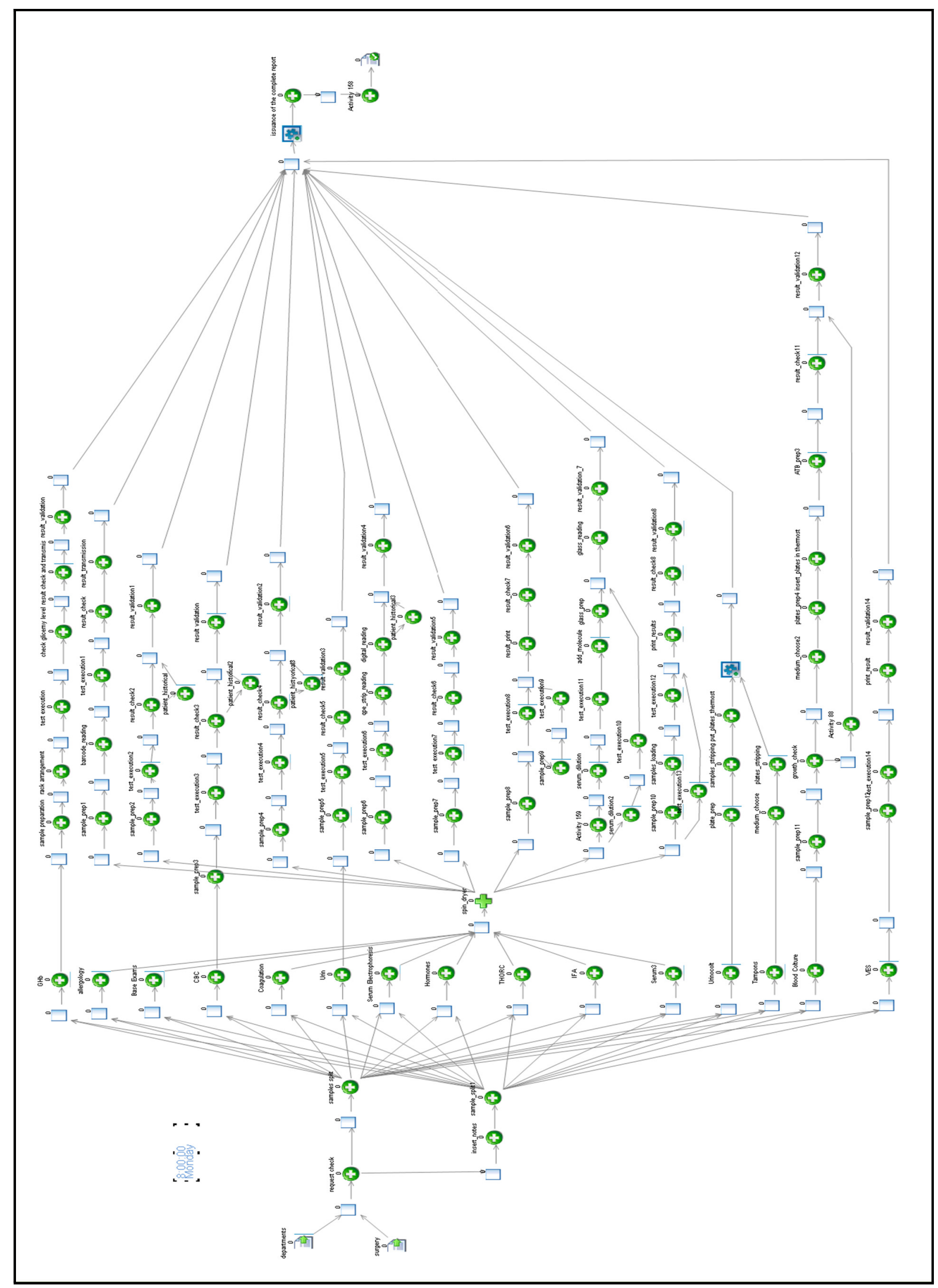

Figure 3. Model of the routine workflow activities. 


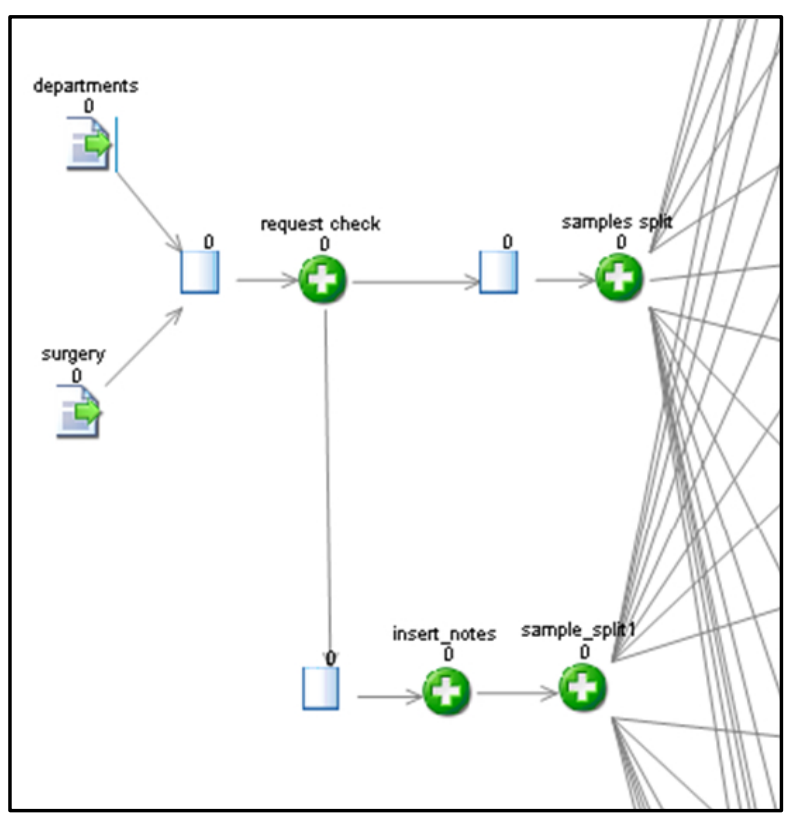

Figure 4. Initial phase and sorting.

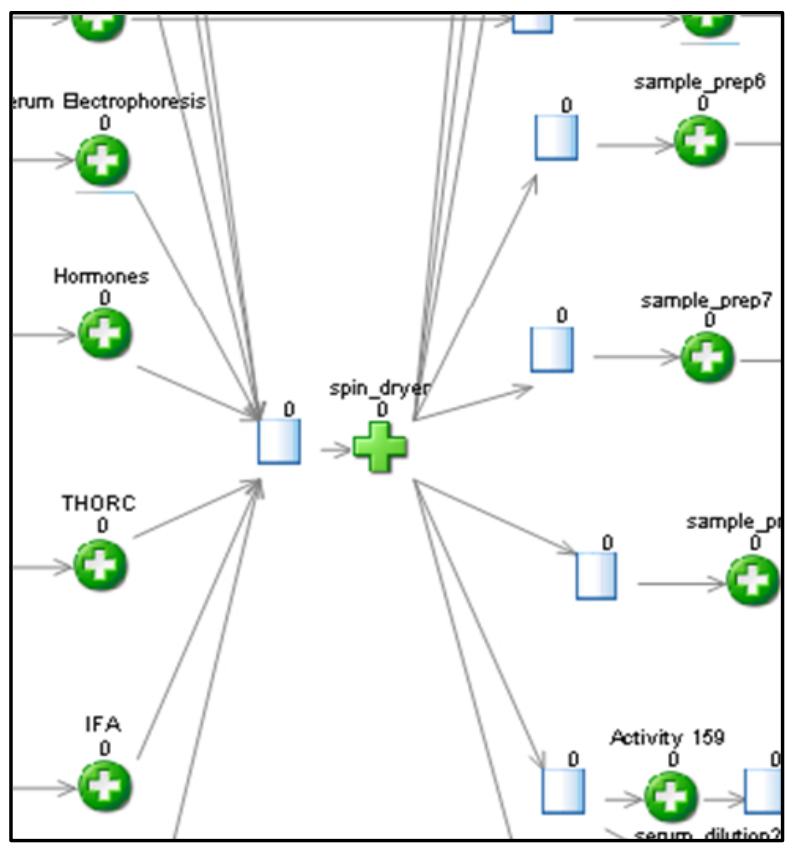

Figure 5. Spin-Dryer phase.

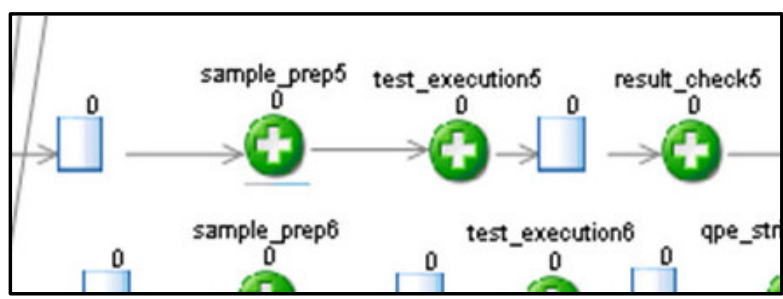

Figure 6. Accumulation of samples and preparation of the samples phases. 
Table $4 . \mathrm{N}^{\circ}$ of samples accumulated and preliminary phases per single exam.

\begin{tabular}{|c|c|c|c|}
\hline Type of Examination & $\mathbf{N}^{\circ}$ of Accumulated Samples & Preliminary Phase & Description of the Preliminary Phases \\
\hline $\mathrm{GHb}$ & 5 & Samples Preparation & The samples are prepared for the exams \\
\hline Allergology & 10 & Samples Preparation & The samples are prepared for the exams \\
\hline $\begin{array}{l}\text { Basic Examinations, } \\
\text { CBC, Coagulation, } \\
\text { Urine }\end{array}$ & $\begin{array}{l}\text { Variable, according to the } \\
\text { number of examinations to } \\
\text { be performed }\end{array}$ & Samples Preparation & The samples are prepared for the exams \\
\hline & & Samples Preparation & The samples are prepared for the exams \\
\hline Urineculture, Swabs & $\begin{array}{l}\text { Variable, according to the } \\
\text { number of examinations to } \\
\text { be performed }\end{array}$ & Plate Preparation & $\begin{array}{l}\text { The samples were plated and placed inside } \\
\text { a thermostat in which they are left for } 24 \\
\text { hours in order to increase the bacterial } \\
\text { colonies if present }\end{array}$ \\
\hline & Variable, according to the & Samples Preparation & $\begin{array}{l}\text { The glass bottles containing the test material } \\
\text { and a plot of growth, are kept in an incubator } \\
\text { to allow further bacterial growth }\end{array}$ \\
\hline Blood Culture & $\begin{array}{l}\text { number of examinations to } \\
\text { be performed }\end{array}$ & Plate Preparation & $\begin{array}{l}\text { The samples were plated and placed inside } \\
\text { a thermostat in which they are left for } 24 \\
\text { hours in order to increase the bacterial } \\
\text { colonies if present }\end{array}$ \\
\hline
\end{tabular}

Table 5. Reporting timing per examination.

\begin{tabular}{lc}
\hline \multicolumn{1}{c}{ Type of Exam } & Reporting Timing \\
\hline Base, Coagulation, CBC, Urine & 1 day \\
Hormonal Tests & $48-52$ hours \\
Allergy Examinations, Serology Examination & 7 days \\
Bacteriology Examinations, Urinoculture, Tampons & 1 week \\
Blood Culture & 2 weeks \\
\hline
\end{tabular}

The formal validation consisted in the evaluation of the architectural characteristics of the simulated model as well as the computing code used to implement it; this type of validation can sometimes be called "software debugging”.

In the structural validation the behaviours of the actual system and the simulated model were compared, through the execution of two different types of check:

- Open Box Validation: the model was shown to all the operators of the structure who verified its functioning, especially to:

o Medical Directors;

o Biologist Managers;

o Lab Technicians;

o Medical Chief.

- Black Box validation: the results of the "As-Is" analysis were compared to a set of data, gained from the real system, to verify the compliance between the simulated system and the actual one, especially:

o Total number of requests for examination;

o Number of requests per exam;

o Total number of tests performed;

o Number of examinations performed, broken down by type of examination;

o Time lapse between the examination requests and tests execution.

The comparison made clear that the simulated model provided a good simulation of the real system, meaning that a suitable representation of the working activities carried out in the DLM was performed. 


\subsection{The "As-Is" Analysis}

After the "open box validation" step, the simulation on the workflow of the "routine activities" was performed for the considered time window. To obtain statistically valid results the simulation was implemented on 395 runs; to define the necessary number of runs to make a trial, a Simul8 Tool was implemented, named Calculator Tool Trials (a trial is a series of sequential executions of a single run). For each run both the total number of samples and the characteristics of a single sample remained the same.

According to the logic of the BPM, an economic analysis of the human resources involved in the DLM activities was performed for the time window considered. The "resource cost per minute" asset was considered for every single subject involved in the working activities; the cost of each resource was then calculated as the sum of the costs per minute, multiplied by the real working time of each resource, and the capital cost-the capital cost being the sum of all the fixed costs paid every month by the structure. The "Medical Chief" made only a supervising activity of the workflow, so the percentage of use of this resource was considered only as a part of the capital cost.

The analysis of the results shows that DES's approach realized a quantitative estimation of the different types of resources involved in the activities of the DLM, leading to reveal the corresponding cost for each category, as well as the real costs related to the use of resources. Along with these, the approach implemented also revealed the utilization percentages of the resources involved in the activities of the DLM.

The possibility to show through this type of analysis the effects of any kind of reorganization dynamic (in terms of resources variation/reallocation, costs and utilization percentages), stands a very important result, especially from a management point of view.

\subsection{The "What-If" Analysis}

After a preliminary confrontation with the actors involved in the study, two experiments (or scenarios) were conducted on the model for the evaluation of the same set of KPIs under different operating conditions, in order to get the most likely response of the system. The scope was to figure out how to maximize the working activities, while keeping acceptable costs, in a new organizational structure.

\subsubsection{Scenario \#1: Increasing the Number of Exams}

In the first experiment, the behaviour of the system was evaluated when demand increases and new resources are added in acceptance. To deal with the increased demand, a minimum of 2, and a maximum of 3 dedicated assets for samples acceptance was considered.

\subsubsection{Scenario \#2: Demand Increasing and Addition of a Technician}

In the second experiment, the increase was evaluated in terms of requests, along with the increase in number of resources - a Clinical Chemistry technician was added to the staff in particular. Under such conditions, no structural changes to the model occurred.

\section{Main Findings and Discussion}

Table 6 shows the comparison between the "As-Is" condition and the two scenarios hypothesized as to the first set of workflow items.

The results highlight that, besides the similar higher number of Completed Jobs for both "What-If” scenarios, the introduction of a new asset made possible to contain the (expected) increase of the Time in System for the requests. The same operation was performed as for the percentage of resources utilization (see Table 7), pointing out that:

- Great part of the resources featured similar values between the two scenarios (e.g. Technician_qpe);

- A small set of resources (Technician_cc, Doctor_cc, Technician_bact) showed instead lower values for scenario \#2.

The increase of resources utilization affected more significantly the scenario \#1: the introduction of the new asset made in fact evident a general positive effect especially for what concerns the CC-related resources, the others showing no differences between the two scenarios.

In Table 8 presents the costs related to the resources, evaluated on the basis of the actual working times of each resource, in every scenario, for the entire length of the simulation run. 
Table 6. Comparison between end point results.

\begin{tabular}{cccc}
\hline End Point & Baseline & BL Vs. Scenario \#1 & BL Vs. Scenario \#2 \\
\hline Number Completed Jobs & 553 & 922 & 926 \\
[\% Variation] & & +66.72694 & +67.45027 \\
Average Time in System (min.) & 369.38 & 528.28 & 447.67 \\
{$[\%$ Variation] } & & +43.01803 & +21.19498 \\
\hline
\end{tabular}

Table 7. Comparison between \% of resources utilization.

\begin{tabular}{cccc}
\hline Resources & Baseline [\%] & BL vs. Scenario \#1 [\%] & BL vs. Scenario \#2 [\%] \\
\hline Technician_cc & 67.70 & 96.29 & 85.04 \\
[\% Variation] & & +42.23 & +25.61 \\
Doctor_cc & 52.74 & 89.57 & 66.28 \\
[\% Variation] & & +69.83 & +25.67 \\
Technician_ba & 37.04 & 51.20 & 49.48 \\
[\% Variation] & & +38.23 & +33.58 \\
Doctor_ba & 18.88 & 29.53 & 29.92 \\
[\% Variation] & & +56.41 & +58.47 \\
Doctor_serology & 40.04 & 61.31 & 61.31 \\
[\% Variation] & & +53.12 & +53.12 \\
Doctor_hormones & 17.63 & 19.92 & 19.92 \\
[\% Variation] & & +12.99 & +12.99 \\
Technician_qpe & 63.03 & 79.55 & 79.55 \\
[\% Variation] & & +26.21 & +26.21 \\
Doctor_qpe & 39.79 & 61.74 & 61.76 \\
[\% Variation] & & +55.16 & +55.21 \\
\hline
\end{tabular}

Table 8. Comparison between costs categories.

\begin{tabular}{|c|c|c|c|}
\hline \multirow{2}{*}{ Cost Category } & Baseline & BL vs. Scenario \#1 & BL vs. Scenario \#2 \\
\hline & \multicolumn{3}{|c|}{ Cost (€) } \\
\hline \multirow[t]{2}{*}{ Total Costs related both to resources number and capital costs } & 23359.55 & 23359.55 & 24754.93 \\
\hline & & 0 & +5.97 \\
\hline Total Costs related to resource utilization & 22050.49 & 22050.07 & 22815.87 \\
\hline [\% Variation] & & -0.0019 & +3.47 \\
\hline Techn_cc Total Cost & 2720.98 & 2838.43 & 3628 \\
\hline$[\%$ Variation $]$ & & +4.32 & +33.33 \\
\hline Doctor_cc Total Cost & 3420.00 & 3143.19 & 3419.82 \\
\hline [\% Variation] & & -8.09 & -0.00526 \\
\hline Tecnh_ba Total Cost & 1824.00 & 1814.88 & 1181.1 \\
\hline [\% Variation] & & -0.5 & -35.25 \\
\hline Doctor_ba Total Cost & 4628.48 & 4828.44 & 5130 \\
\hline [\% Variation] & & +4.32 & +10.83 \\
\hline Doctor_serology Total Cost & 1710.00 & 1710 & 1709.95 \\
\hline [\% Variation] & & 0 & -0.00292 \\
\hline Doctor_hormones Total Cost & 3420.00 & 3355.72 & 3420 \\
\hline [\% Variation] & & -1.88 & 0 \\
\hline Tecnh_qpe Total Cost & 907.03 & 919.42 & 907 \\
\hline [\% Variation] & & +1.36 & -0.00331 \\
\hline Doctor_qpe Total Cost & 3420.00 & 3439.99 & 3420 \\
\hline [\% Variation] & & +0.58 & 0 \\
\hline
\end{tabular}


It was possible to verify how the percentage fluctuations appeared as more limited in scenario \#1 than in scenario \#2. More in particular, the variation that induced the first "What-If" analysis substantially left the rest of the workflow unaltered in terms of assets involved; the most evident variations (e.g. Doctor_cc and Techn_cc) pointed out that the simple increase of requests on the one hand lowers the working burden for the Doctor in the Clinical Chemistry Section, while on the other hand increases accordingly the tasks for the Technicians. The increased number of completed works also caused a slight increase for the doctors in the Bacteriological Section. The new technician for the Chemical Chemistry Section clearly influenced the cost increase for the category; also in this case, the higher number of completed activities affected the Bacteriological Section, bringing an augmented workload for the Doctors, and highly reducing the working burden for the technicians.

As for the other categories, small differences resulted between the baseline and the two scenarios; in some cases (e.g. Doctor_serology) the use of many significant figures resulted from the simulation was just intended to show the preciseness of the method adopted, nonetheless it was possible to consider the two working dynamics as just alike.

In the end, the simulation made it possible to figure out the main factors affecting the costs variation between the scenarios, especially: 1) number of resources implemented; 2) number and type of exams; 3) time spent for each single activity. Table 9 and Table 10 focus on the costs analysis via different perspectives.

The number of IFA exams in both scenarios was set equal to zero, since those would be no longer performed in the "San Paolo" Hospital. This type of exam was not evaluated then for the analysis of the variation of the costs.

Both "What-If" scenarios, introducing elements to improve the process dynamics, induced the same high increasing of the number of exams, which implied in turn the formation of small economies of scale (given the limited amount of time considered for the simulation). More interesting was the analysis of the percentage decrease of the costs, which showed once more how the introduction of a new asset largely affected the workloads among the different categories involved, making the work line somehow "lighter" and allowing as a consequence on the medium/long term savings greater than the costs to be supported to pay the asset itself.

Table 9. Comparison between costs analysis per exam.

\begin{tabular}{|c|c|c|c|c|c|c|}
\hline \multirow{2}{*}{ Cost Category } & \multicolumn{2}{|c|}{ Baseline } & \multicolumn{2}{|c|}{ Scenario \#1 } & \multicolumn{2}{|c|}{ Scenario \#2 } \\
\hline & N. Exams & Cost (€) & N. Exams & Cost (€) & N. Exams & Cost $(€)$ \\
\hline $\mathrm{GHb}$ & 15 & 4.1 & 25 & 3.93 & 25 & 3.93 \\
\hline Allergology & 10 & 5.33 & 10 & 5.29 & 10 & 5.28 \\
\hline Base Exams & 426 & 3.26 & 755 & 3.26 & 755 & 3 \\
\hline $\mathrm{CBC}$ & 358 & 2.84 & 634 & 2.73 & 634 & 2,61 \\
\hline Coagulation & 190 & 3.97 & 331 & 3,89 & 331 & 3.73 \\
\hline Urine & 179 & 3.24 & 309 & 3.19 & 309 & 3.19 \\
\hline Serum Electrophoresis & 180 & 8.86 & 297 & 8.81 & 300 & 8.8 \\
\hline Hormones & 132 & 1.59 & 180 & 1.57 & 180 & 1.54 \\
\hline THORC & 10 & 20.4 & 30 & 17.03 & 30 & 17.03 \\
\hline IFA & 10 & 25 & 0 & 0 & 0 & 0 \\
\hline Serum3 & 10 & 17.13 & 30 & 16.22 & 30 & 16.22 \\
\hline Urino Cultures & 39 & 12.95 & 63 & 12.9 & 63 & 10.79 \\
\hline Tampons & 3 & 15.34 & 5 & 13.08 & 5 & 13.01 \\
\hline Blood Cultures & 2 & 28.58 & 2 & 28.55 & 3 & 20.79 \\
\hline VES & 22 & 2.73 & 33 & 1.21 & 33 & 1.08 \\
\hline Total Cost Related to Exams & \multicolumn{2}{|c|}{6952.81} & \multicolumn{2}{|c|}{11488.37} & \multicolumn{2}{|c|}{11048.66} \\
\hline
\end{tabular}


Table 10. \% variation of the costs of exams.

\begin{tabular}{ccc}
\hline Cost Category & \multicolumn{2}{c}{ \% Variation of Costs } \\
GHb & BL vs. Scenario $\# \mathbf{1}$ & BL vs. Scenario \#2 \\
Allergology & -4.15 & -4.15 \\
Base Exams & -0.75 & -0.94 \\
CBC & 0 & -7.97 \\
Coagulation & -3.87 & -8.09 \\
Urine & -2.01 & -6.04 \\
Serum Electrophoresis & -1.54 & -1.54 \\
Hormones & -0.56 & -0.68 \\
THORC & -1.26 & -3.14 \\
Serum3 & -16.52 & -16.52 \\
Urino Cultures & -5.31 & -5.31 \\
Tampons & -0.39 & -16.68 \\
Blood Cultures & -14.73 & -15.19 \\
VES & -0.11 & -27.26 \\
\hline
\end{tabular}

\section{Conclusions}

This work is part of a larger program aimed at formalizing a specific systems reengineering methodology, with the main purpose to improve the studies on healthcare organizations' internal workflows. Healthcare provides a fertile ground for the development of new modelling approaches, capable of blending the exigencies from both the communities of business administration and computer science. Under such perspective, in fact, the adoption of any form of technology, especially for what refers to the healthcare sector, requests to find out in the first place whether or not it: 1) is actually accessible from the ones who need it (availability); 2) can lead to a better health status (efficacy); 3) can be measured through only one "natural" parameter, so to get to an easier assessment between two or more alternatives (cost-efficacy); 4) actually affects patients' everyday habits (effectiveness); 5) comes with sustainable costs (cost-effectiveness).

For what concerns the approach implemented in the present study: 1) the use of simulation grants the right access to the outcomes of a BPM-related logic for all the professionals involved; 2) the ex-ante evaluation of the most timely benefits is closely related to the positive impact of the system on the whole DLM internal functioning; 3) the possibility of a multiple choice among the KPIs to work on with can lead to work out as many "What-If" scenarios as requested from the actors interested in the simulation; 4) developing plausible scenarios has the main purpose to get to better conditions through which improve the quality of care; 5) a properly performed DES/BMP approach can be seen as an advanced form of robust design, so that the initiative costs will be carefully sized since the beginning.

The most innovative aspect concerning the use in the healthcare context of a methodology capable of combining both DES potentialities and BPM logics, relates eventually to the possibility to consider, quickly and with minimal cost, the efficiency and the effectiveness of the proposed changes without altering the dynamics of the real system: an evolutionary step for the process analysis.

\section{References}

[1] Tamburis, O., Ricci, F.L. and Pecoraro, F. (2014) A Mathematical Model to Plan the Adoption of HER Systems. In: Wang, J., Ed., EBAO_Encyclopedia of Business Analytics and Optimization, IGI-Global, Hershey, 14-29.

[2] Torri, A. and Pepino, A. (2013) Use of New Management Techniques in the Italian Health Care System. Scholars Journal 
of Engineering and Technology, 1, 195-197.

[3] Tähkäpää, J. (2004) Increasing Role of Information Systems in Public Health Care-Challenge to the Management. Proceedings of the 37th Hawaii International Conference on System Sciences, Hawaii, 5-8 January 2004.

[4] Torri, A., Pepino, A., Rovani, M. and De Luca, N. (2014) Use of DES for the Quantitative Analysis of the Workflow of the Hypertension Center at the "Azienda Ospedaliera Universitaria Federico II" of Naples. International Journal of Computer Theory and Engineering, 6, 220-226. http://dx.doi.org/10.7763/IJCTE.2014.V6.866

[5] Wang, B., McKay, K., Jewer, J. and Sharma, A. (2013) Physician Shift Behavior and Its Impact on Service Performances in an Emergency Department. Proceedings of the 2013 Winter Simulation Conference, Washington DC, 8-11 December 2013, 2350-2361. http://dx.doi.org/10.1109/WSC.2013.6721610

[6] Torri, A., Rovani, M., Abbate, T. and Pepino, A. (2013) Performance Evaluation in a Department of Laboratory Medicine. Proceedings of the 2013 Winter Simulation Conference, Washington DC, 8-11 December 2013, 3972-3973. http://informs-sim.org/wsc13papers/by_area.html.

[7] Kotiadis, K., Tako, A.A. and Vasilakis, C. (2014) A Participative and Facilitative Conceptual Modelling Framework for Discrete Event Simulation Studies in Healthcare. Journal of the Operational Research Society, 65, 197-213. http://dx.doi.org/10.1057/jors.2012.176

[8] Kabene, S.M., Orchard, C, Howard, J.M., Soriano, M. A. and Leduc, R. (2006) The importance of Human Resources Management in Health Care: A Global Context. Human Resources for Health, 4, 20. http://dx.doi.org/10.1186/1478-4491-4-20

[9] Lim, E.M., Worster, A., Goeree, R. and Tarride, J.E. (2013) Simulating an Emergency Department: The Importance of Modeling the Interactions between Physicians and Delegates in a Discrete Event Simulation. BMC Medical Informatics and Decision Making, 13, 59. http://dx.doi.org/10.1186/1472-6947-13-59

[10] Banks, J., Carson II, J.S., Nelson, B.L. and Nicol, D.M. (2009) Discrete-Event System Simulation. 5th Edition, Prentice Hall, Upper Saddle River.

[11] Jacobson, S.H., Hall, S.N. and Swisher, J.R. (2006) Discrete-Event Simulation of Health Care Systems. Patient Flow: Reducing Delay in Healthcare Delivery International Series in Operations Research \& Management Science, 91, 211-252. http://dx.doi.org/10.1007/978-0-387-33636-7_8

[12] Victor, P., Andreev, V.P., Head, T., Johnson, N., Deo, S.K., Daunert, S. and Goldschmidt-Clermont, P.J. (2013) Discrete Event Simulation Model of Sudden Cardiac Death Predicts High Impact of Preventive Interventions. Scientific Reports, 3, Article Number: 1771, 1-8.

[13] Kim, B., Elstein, Y., Shiner, B., Konrad, R., Pomerantz, A.S. and Watts, B.V. (2013) Use of Discrete Event Simulation to Improve a Mental Health Clinic. General Hospital Psychiatry, 35, 668-670. http://dx.doi.org/10.1016/j.genhosppsych.2013.06.004

[14] Rau, C.L., Tsai, P.F., Liang, S.F., Tan, J.C., Syu, H.C., Jheng, Y.L., Ciou, T.S. and Jaw, F.S. (2013) Using Discrete-Event Simulation in Strategic Capacity Planning for an Outpatient Physical Therapy Service. Health Care Management Science, 16, 352-365. http://dx.doi.org/10.1007/s10729-013-9234-2

[15] Van der Aalst, W.M.P., ter Hofstede, A.H.M. and Weske, M. (2003) Business Process Management: A Survey, Lecture Notes in Computer Science. Proceedings of the 2003 International Conference on Business Process Management, Eindhoven, 26-27 June 2003, 1-12.

[16] www.simul8.com. 